\title{
Proofs Versus Experiments: Wittgensteinian Themes Surrounding the Four-Color Theorem
}

\author{
Gisele Dalva Secco and Luiz Carlos Pereira
}

\section{Introduction}

The publication of the Four-Color Theorem proof (4CT proof) can be seen as the closure of a mathematical history involving above all topics in topology, combinatorics and graph theory. It can also be considered as the turning point in the historical relations between mathematics and computer science, especially with respect to the use of computer machinery to establish original mathematical results - it is broadly known that the main reason why this proof provoked a certain commotion in the mathematical community is the indispensable participation of computers in its construction.

Even though the case of the 4CT proof is referred in the literature mostly as the historically remarkable turning point that it in fact is, it is also a source of interesting conceptual distinctions, related to old and new philosophical questions. ${ }^{1}$ The aim of this paper is to present some of these distinctions and questions, especially those which can be approached from a wittgensteinian perspective, such as the distinction between proofs and experiments and the possibility of a sort of functional reading

\footnotetext{
${ }^{1}$ Mentions to the 4CT proof can be found in Avigad (2008) and McEvoy (2013). For more detailed approaches considering new philosophical questions on the role of computers in mathematical practices, see MacKenzie (2001, 2005), and for old questions revisited see Bassler (2006), McEvoy (2008, 2013) and Prawitz (2008).

G.D. Secco $(\square)$

Federal University of Rio Grande do Sul, Porto Alegre, Brazil

e-mail: gisele.secco@ufrgs.br

L.C. Pereira

Pontifical Catholic University of Rio de Janeiro, Rio de Janeiro, Brazil

e-mail: luiz@inf.puc-rio.br
} 
of propositions proved with the assistance of computers. In the next section we shall present the main ideas of the 4CT proof, the four claims that provoked the philosophical discussion about it and the main points of this discussion. Section three analyses how the wittgensteinian distinction between proofs and experiments was suggested in the course of those philosophical disputes, and the fourth section proposes a complementary way of projecting the distinction into the debate, and concluding that some alleged problems can be dissolved in another wittgensteinian light.

\section{The Four-Color Theorem Proof and Four Philosophical Claims}

The 4CT states that every map under certain circumstances (normal planar maps) can be painted with just four colors without any adjacent region being painted with the same color - which amounts to say that every such a map is admissibly fourcolorable. ${ }^{2}$ The proof of this statement was presented in a pair of papers written by Appel and Haken (1977a) and Appel et al. (1977), and it can be considered as the conclusion of a history initiated 125 years before with a guess about the possibility of coloring geographical maps with the restriction to four-colorability. ${ }^{3}$

Since the first attempt to prove the 4CT by A. B. Kempe in 1879, until the more recent ones, ${ }^{4}$ the general structure of the proof is that of a reductio ad absurdum in which the initial assumption affirms the existence of a map demanding five colors to be admissibly colored and also that this is the smallest of such maps, i.e., a minimal five-chromatic normal map. The reductio includes a proof by cases in which the application of the so-called reducibility methods permits the construction a set of configurations such that every five-chromatic map has to contain at least one of these configurations. This set is called the unavoidable $U$. It is then possible to show that the initial map is not the smallest five-chromatic normal map it was assumed to be. Appel, Haken and Koch's results show that construction of the unavoidable set of reducible configurations that contradicts the initial assumption of the reductio is obtained not with only four cases, as Kempe supposed in his alleged proof, but

\footnotetext{
${ }^{2} \mathrm{~A}$ normal planar map is a map on the plane in which there is no region completely surrounded by other and there are no more than three regions connecting in the same point. The topological version of the 4CT affirms: "For every map there exists an admissible 4-colouring"; the combinatorial one, without any reference to geometry or topology: "Every planar graph has an admissible vertex 4-coloring" (Fritsch and Fritsch 1998).

${ }^{3}$ The guess was made by a Geography teacher whose brother was a student of Augustus de Morgan. It was De Morgan who first brought mathematical attention to the problem in letters to Hamilton (from 1852 to 1853) and in a review of William Whewell's The Philosophy of Discovery published in 1860. For details about the history of the different attempts to solve the problem, besides Fritsch and Fritsch 1998, see Saaty and Kainen 1986, MacKenzie 2001 and Wilson 2002.

${ }^{4}$ Robertson et al. 1997 and Gonthier 2008.
} 
with more than a thousand subcases that are practically impossible to be constructed without the execution of computer programs.

From the beginning, reactions to the result within the mathematical community were of two kinds. ${ }^{5}$ On the one hand, some criticisms were driven to the apparent lack of structure (and elegance) of the proof - an aspect directly related to its combinatorial nature. On the other hand, graph theorists claimed that the kind of (combinatorial) complexity involved in the 4CT proof does not notably differ from the complexity of other proofs in the field. ${ }^{6}$ In the first group are those who could not accept computer-assisted proofs - especially this one, given the impossibility of human verification of each calculatorial step of its computational aided part and the subsequent possibility of an error in the procedure. The second group includes defendants of the use of computers in mathematical practices, with no serious concerns about the "urban legend" of hidden errors in the programs. ${ }^{7}$

Dissatisfaction with the result was also manifested in the field of computer science, for the programs executed to realize the necessary calculi to establish the key lemma of reducibility were not formally verified. This criticism is related to those just mentioned, in so far as the formal verification of the programs could immunize the computational procedure from errors. Given the aims of this paper it is interesting to note the kind of argument the authors engendered in responding to this sort of criticism:

Most mathematicians who have some computer background would be satisfied with a copy of the program, some way of verifying that the inputs were typed in correctly, and some

\footnotetext{
${ }^{5}$ Appel said (see MacKenzie 2001: 138) that in one of the first public briefings of the result the audience had been clearly divided into two groups: people with more than 40 years that "could not be convinced that a proof by computer could be correct" and "people under forty [who] could not be convinced that a proof that took 700 pages of hand calculations could be correct". Appel was referring to a talk given by Haken's son at Berkeley, who at that time (1977) was a graduate student at the UCB and gave this talk to announce and describe the proof in that institution.

${ }^{6} \mathrm{~A}$ topic not explored here is the connection between the alleged lack of structure of the computational part of the proof and the absence of explanatory virtues in it (a sample of this kind of criticism can be found in Ian Stewart's Concepts of Modern Mathematics (Stewart 1995: 304)). Nevertheless, Swart argues that if the proof of the 4CT is not explanatory, then no proof by exhaustion is. They can be divided into three parts: "(i) Establishing the fact that the theorem is true provided a certain set of graphs, configurations, or - in general - cases possesses (or do not possess, as the case may be) a stated property; (ii) Obtaining an exhaustive listing of these cases; (iii) Confirming that all the members of this set do possess the required property. The finite set of cases concerned may, at one extreme, be so small and so simple that the case testing can be done in our heads, or it may, at the other extreme, be so large and/or so complicated that it is impossible to carry out without the help of a computer" (Swart 1980: p. 699).

${ }^{7}$ In fact, during the 1980s, a student of electric engineering, Ulrich Schmidt, found an error that could be considered relevant in the proof - being "relevant" an error that could interfere in the final result of the derivation. Nevertheless, Appel and Haken not only developed an "error-correcting routine" (Appel and Haken 1986: 19-20), but also presented a typology of errors according to which the main error found by Schmidt wasn't relevant. The authors attribute the rumours surrounding the correctness of the proof to "a misinterpretation of the results of the independent check of details of the proof by U. Schmidt" (Appel and Haken 1986: 10).
} 
output that indicated that the program ran to its end. Any reader who remained worried could easily program a matrix multiplication algorithm for his or her own computer, type in the inputs and run a check program. What qualms remain would very likely result from the possibility that both author and checker had misread some of the input entries, but certainly this is less worrisome than the difficulties involved in performing similar tasks by hand. I would call such a proof an easily replicable proof. (Appel 1984: 35, our emphasis)

In the last section of the paper we will return to Appel and Haken's responses in order to show that they can be used to retort not only to mathematical but also to philosophical criticisms. By now, let us only stress that the passage above presents a sort of rhetorical-dialectical argument ${ }^{8}$ : if the audience is sufficiently familiarized with the way in which computers worked (late 1970s computers, of course), being able to survey the algorithm for the calculations, they could so to speak have control over the calculations, in the sense that they could survey the algorithm running the calculations.

In the philosophical world reactions to the 4CT proof began even before its publication, ${ }^{9}$ but it seems fair to say that the philosophical citizenship of this famous mathematical result is due to an argument presented by Thomas Tymoczko (Tymoczko 1979), the introduction of the experiments in mathematics argument (IEA). The "four philosophical consequences" mentioned in the title of this section are, in fact, the consequences Tymoczko extracts from the IEA. They are all statements related to an alleged need to abandon or modify "many commonly held beliefs about mathematics", such as:

C1. All mathematical theorems are known a priori.

C2. Mathematics, as opposed to natural science, has no empirical content.

C3. Mathematics, as opposed to natural science, relies only on proofs, whereas natural science makes use of experiments.

C4. Mathematical theorems are certain to a degree that no theorem of natural science can match. (Tymoczko 1979: 63)

These claims and their negations articulate traditional conceptual distinctions, such as (C1) a priori versus empirical knowledge, (C2) mathematics versus natural sciences, $(\mathrm{C} 3)$ the distinction between proofs and experiments, and (C4) the idea that the kind of certainty produced by mathematics is of the highest degree. Given that every discussion on "the use of computers in mathematics", from the early $1980 \mathrm{~s}^{10}$ until today, ${ }^{11}$ not only mentions but also reacts against some point (either premises or conclusions) of Tymoczko's IEA, any narrative on how wittgensteinian

\footnotetext{
${ }^{8}$ The idea that mathematical practices have a rhetorical aspect is being explored, for example, by Gionavva Cifoletti (cf. Cifoletti 2006). See also Lassalle Casanave 2008.

${ }^{9}$ Georg Kreisel (Kreisel 1977) and Hao Wang (Wang 1981) were the first members of the philosophical community that mentioned the 4CT proof in 1977. They knew about the result, respectively, by means of an article published in Scientific American (Appel Haken 1977b) and an expository paper published in New Scientist (Appel 1976). Wang (1981) is the register of a series of talks given by the author in the Chinese Academy of Science in 1977.

${ }^{10}$ The most cited ones being Detlefsen and Luker 1980 and Teller 1980. See also Swart (1980).

${ }^{11}$ Besides the bibliography indicated in footnote 1 see Turner Eden (2017) and Baker (2016).
} 
topics entered into the arena of debates about the 4CT proof depends on an adequate reading of Tymoczko's argument and its reception. We present below a very short version of our reading the argument.

The starting point of the IEA is the observation that the 4CT proof is neither constructible nor verifiable in the traditional way by one single person in a lifetime. The argument then associates this fact with the essential use of computers (as instruments similar to those used in scientific experiments), concluding that it is necessary to revise the standard conception of mathematical proof in such a way that the revised conception can incorporate in mathematics the possibility of error, a characteristic of experimental procedures. Our reconstruction of Tymoczko's IEA is as follows:

$(\alpha)$ The major features of mathematical proofs, traditionally considered as "a priori deduction of a statement from premises" (Tymoczko 1979: 58), are: $\left(\alpha_{\mathrm{a}}\right)$ proofs convince, $\left(\alpha_{b}\right)$ proofs are surveyable e $\left(\alpha_{c}\right)$ proofs are formalizable;

( $\beta$ ) The 4 CT proof does have features $\left(\alpha_{a}\right)$ and $\left(\alpha_{c}\right)$, but it doesn't have feature $\left(\alpha_{b}\right)$, since the calculi executed with computational aid cannot be verified by a person in a lifetime;

$(\gamma)$ Given that the our confidence in the machines rests "on the assessment of a complex set of empirical factors" (Tymoczko 1979: 74), the use of computers in proofs introduces experiments in the mathematical domain;

( $\delta$ ) Hence, by appealing irrevocably to the execution of computer programs, the 4CT proof "makes the 4CT the first mathematical proposition to be known $a$ posteriori". Mathematics is then subject to the same kind of error that is typical of experimental methodologies, what forces us to change "the sense of the underlying concept of "proof'" (Tymoczko 1979: 58).

Even if it is not our aim here to present a detailed analysis of how Tymoczko achieves those four philosophical consequences from his IEA, we must stress that the aforementioned traditional conceptual distinctions, as well as the crucial notion of surveyability, are not clearly used in the IEA. According to $(\beta)$, for example, surveyability is a missing aspect of the 4CT proof, for "no mathematician has seen a proof of the 4CT, nor has any seen a proof that it has a proof." (Tymoczko 1979: 58) But what does it mean to say that no mathematician saw the 4CT proof?

Tymoczko uses the expressions to see, to verify and to survey in a very elastic way. Sometimes he seems to understand that to survey corresponds to a general inspection, identifying surveyability with the property proofs have to be visualized at a glance - in such a way that Appel and Haken's work could be considered as a counterexample to the "traditional" concept of proof - while at times he takes surveyability as the ability to carry out a step-by-step verification, an algorithmic check of the entire proof. He also identifies this feature with the possibility of the manual realization of the calculi involved in the proof of the key lemma that produces the unavoidable set $U$. Even with this kind of semantic fluctuation, the author tenaciously insists in associating the impossibility of a step-by-step verification - "no computer has printed out the complete proof of the reducibility lemma" (Tymoczko 1979: 68) - with the alleged empirical character and the 
possibility of error in the procedure. Curiously, the immediate recognition of the fact that "such a printout [wouldn't] be of much use to human mathematicians" (loc. cit.) seems to indicate that, according to Tymoczko, the second sense of surveyability does not contribute to the first one.

The distinction between local and global surveyability suggested by Bassler (2006) could be put to good use here. While local surveyability corresponds to the capacity of algorithmic verification, global surveyability is related to the possibility of grasping the sufficiency of the steps (the rules) for proving the theorem. We could then say that the lack of local surveyability of the 4CT proof in Tymoczko's IEA implies the recognition of its empirical character - an argumentative move whose plausibility depends on the clarification of the notion of calculus or computation. But this clarification is not found in Tymoczko's paper, and only timidly in the responses to his IEA.

One way of presenting the controversies about the IEA, which we borrow from Dag Prawitz, ${ }^{12}$ consists in dividing the scenario in which they took place into two groups: those who affirm and those who deny the occurrence of any significant change in mathematical practices with the advent of the 4CT proof. Tymoczko is situated in the first group. The second group is also divided into two subgroups: those who do not accept that allegedly empirical procedures - such as the use of computers in the proof of the 4CT - have any meaningful role in proofs (Teller 1980) and those who believe that such procedures can be meaningful but do not constitute any such novelty in mathematics (Detlefsen and Luker 1980). For Detlefsen and Luker, calculating procedures, being executed by humans or machines, do have empirical aspects and there is no problem in accepting partially empirical grounds for proofs - especially for such heavily computational proofs such as the 4CT proof. For Teller, Tymoczko confuses the proof with the verification of the proof, claiming that the novelty in the case of the $4 \mathrm{CT}$ proof lies in the verification process. Here is how he makes his point:

Tymoczko has not given us any reason to suppose these [very complicated proofs] not to be perfectly good proofs in the usual sense. If a computer is programmed to use the same methods of proof we use, a proof that it produced would be a proof in our old sense. Legitimate worries whether an error might have been made only show that there may be legitimate worries whether the proof is correct, not that the proof is a proof only in some new sense even if no mistakes have been made. To put the point in a very slightly different way, the fact that I cannot follow a complex proof produced by a good mathematician does not show that such a mathematician's complex proof is a proof in a different sense of the word from a proof that I can follow. In the same way, the fact that no mathematician may be able to follow a proof produced by a computer does not show that such a computer-produced proof is a proof only in some new sense. (Teller 1980: 800)

Note that we can again use Bassler's distinction between local and global surveyability to stress that when Teller talks about no mathematician being able "to follow" the proof, he is talking about local surveyability. Besides, any error we could be afraid of being committed in a computer-assisted proof could not be appointed

\footnotetext{
${ }^{12}$ Prawitz 2008. For a more critical approach on Prawitz's position see Secco (2015).
} 
as the source of the invasion of empirical elements in mathematics. In resume, the group denying the relevance of the novelty represented by the 4CT proof does not accept Tymoczko's thesis about the need of revising claims C1-C3, either because empirical aspects are not mathematically relevant to the proof of the 4CT or because they are present even in proofs whose execution and reproduction do not depend on computer machines. With respect to the lack of surveyability the rejoinders seem to share the idea that it has nothing to do with the dichotomy a priori versus $a$ posteriori evidence and knowledge, mitigating then the consequences claimed by Tymoczko.

In order to partially complement this sketchy panorama à la Prawitz, we should give an account of Prawitz's own analysis of the case. Given his defense of the thesis that the grounds we have to know the 4CT are partially empirical, one could say that Prawitz sides with Tymoczko. Nevertheless, the similarities between Tymoczko's and Prawitz's conclusions can only be correctly stated with the aid of some nuances. One specificity of Prawitz's approach is that he never proposes, like Tymoczko does implicitly in $(\gamma)$ and $(\delta)$, and explicitly in the negation of claim $C 4$, that mathematics has practically lost its special position into the classification of sciences because of the possibility of error lurking in the proof of the key lemma of the 4CT proof. Rather, even though Prawitz agrees with Tymoczko that

If a theorem has been established only by relying on computers as in the proof of the fourcolour theorem or in a proof that involves big computations, then the proof is not entirely deductive, and there is the undeniably epistemological consequence that the theorem is known only a posteriori. (Prawitz 2008: 92)

He makes an effort to distinguish between the epistemological consequence just mentioned - in which he agrees with the negation of claim $\mathrm{C} 1$ proposed by Tymoczko - and the question about the (supposed lack of) confidence in the partially empirically grounded deductive procedures of the machine, related to Tymoczko's negation of claim $\mathrm{C} 4$. He affirms that it is not unusual to come upon a situation where a "computer report of having found a specific proof may rightly be deemed as very trustworthy and as more trustworthy than a corresponding report from a human of having found a deductive proof" (Prawitz 2008: 92-93). Compared with Tymoczko's idea that the result was a kind of hybrid between proof and experiment in virtue of the "calculatorial gap" that was filled by an unsurveyable experiment (the running of the calculi in computer machines), Prawitz's perspective on the 4CT is much subtler and certainly detached from the anti-foundationalist intentions we can recognize in Tymoczko's paper. We can also say that the conceptual questions emerging from Prawitz's approach have interesting points in common with other discussions about the use of computers in mathematical proofs. ${ }^{13}$

\footnotetext{
${ }^{13}$ Prawitz deals with cases of proofs produced by computers programs - the $4 \mathrm{CT}$ proof being his main example - and proofs that verify programs. Investigating the sociology of mathematical proofs MacKenzie (2005) also considers two categories: proofs using computers and proofs about computers. Each category contains different dimensions: in the first one, automated theorem provers and model checkers, mathematical proofs of immense complication of detail and, in
} 
Some of these questions are clearly related to a long tradition of conceptual dichotomies in philosophy that seeks to contrast two semantic, logical, metaphysical and epistemological fields. The most important examples in this long tradition are: [D1] Leibniz's distinction between truths of reason and truths of fact, [D2] the Humean distinction between relations of ideas and matters of fact and [D3] the Kantian distinction between analytic judgments and synthetic judgments, and between a priori knowledge and a posteriori knowledge. One is the field of necessity, analytic connections and independence of experience; the other is the field of contingency non-analytic connections and dependence on experience. In some cases, these fields are not connected, in other bridges are created and allow a connection between them. This is the case of Kant with his notion of synthetic a priori judgments, and, more recently, Saul Kripke, with new and apparently more general crossings, the contingent a priori truths and the necessary a posteriori truths. The last connection is of special interest to us, since Kripke explicitly mentions the possibility of the use of computers to establish mathematical statements. The proof vs. experiment dichotomy is now part of this long tradition and we will see in the next section how this dichotomy under a wittgensteinian perspective can shed new light on the relations between these two fields.

\section{Proofs Versus Experiments: A Wittgensteinian Invention Enters the Scene}

In order to finish the panorama just sketched showing how Wittgenstein's ideas about proofs and experiments - one of his own versions of that traditional dichotomies - explicitly entered into the arena of discussions on the 4CT proof, let us present how Stuart Shanker defends an even more drastic conclusion than those Tymoczko claimed to follow from Appel and Haken's result. Maintaining that Tymoczko's argument "had touched on a raw nerve", Shanker claims, in a wittgensteinian vein, that proofs and experiments are categorically distinct procedures. Hence, to conclude for the introduction of experiments in mathematics would be nothing more than a grammatical transgression, even though Tymoczko's IEA departs from a "staunchly Wittgensteinian premise that proof must be surveyable" (Shanker 1987: 130). The seemingly wittgensteinian razor of Shanker ends up suppressing both the procedure (the 4CT proof) and its result (the 4CT) from the domain of mathematical practices. In other words, there is no proof of the Four Color Theorem, just an empirical solution to the Four Color Problem:

[W] hat we are offered is not a proof for the existence of the unavoidable set of reducible configurations, but rather, a description of the experimental procedure that was used to

the universe of artificial intelligence, the question about the possibility of a computer be 'an artificial mathematician' (MacKenzie 2005: 2336); the second category deals with key aspects of microprocessors and computer systems on which lives and national security depend. 
discover it. It is for this reason that Appel-Haken solution of the four-colour problem is empirical rather than mathematical, and hence, that it makes no sense to speak of Appel and Haken 'proving' the 'four-colour theorem' let alone of their solution forcing us to modify our understanding of the concept of proof and theorem. (Shanker 1987: 157)

Shanker sustains this conclusion arguing that, by delegating to computers the calculating tasks involved in 4CT proof, we lost the access to the normative liaisons at stake in it. He exemplifies this kind of relation initially referring to the tractarian concern with "the relation of a number to the law which generates the series in which it occurs. It is the law governing the expansion of the series, not the actual expansion of the series, what must be surveyable." (Shanker 1987: 128) It is known that the early Wittgenstein elaborated an intensional conception of mathematics in which the totality of numbers is not understood as a totality of objects but instead as properties of a formal series. But how could this example be associated with the 4CT proof? Shanker does not give a direct answer to this question; rather, he introduces an association via an example (extracted from the intermediate period of Wittgenstein's thought):

\footnotetext{
Wittgenstein argued that the colour octahedron must be surveyable in the sense that the logical articulations forged by the grammatical construction are perspicuous. Likewise, a proof must be surveyable in the sense that we can grasp the 'law' forged by the proof: 'I must be able to write down a part of the series, in such a way that you can recognise the law. That is to say, no description is to occur in what is written down, everything must be represented' (PR §190). But this is precisely the condition which the Appel-Haken solution fails to meet: what we are given just is a description of $U$ - together with the operations which the computer has performed to test its reducibility - rather than a 'manifestation of the law' for the generation of the unavoidable set of reducible configurations. (Shanker 1987: 153)
}

According to Shanker's reading of Wittgenstein, a mathematical proof is surveyable when the logical, conceptual or normative relations between its steps are graspable in the same way they are surveyable in the wittgensteinian model, the color octahedron. This requirement is clearly used not only to affirm that the 4CT proof does not fit Wittgenstein's considerations about the surveyability of proofs, but also to conclude that Appel and Haken's procedure is experimental and not mathematical as we saw above (Shanker 1987: 157). The main problem with this strategy is indicated by Shanker himself: he recognizes that when Wittgenstein used the notion of surveyability to talk about the grammar of color words (in the Philosophical remarks) he was referring to the idea that the grammatical rules of a system of propositions must be "fully surveyable", meaning that "One should be able to tell simply by looking at a proper model of the grammar of colourwords which moves are allowed in the Satzsystem (PR §222)" (Shanker 1987: 123). But, according to Shanker, when Wittgenstein approaches the question of surveyability of proofs in the late 1930s (in the Remarks on the Foundations of Mathematics), he "made no reference to this early suggestion that it is possible to construct a concretized model of the grammar of a Satzsystem" (loc. cit.), noting that in this period, "he adhered to the idea that the grammatical conventions constructed by a notation must in some sense be phenomenologically surveyable if they are 
to be intelligible" (loc. cit.), Shanker's analysis of the 4CT case associates this "phenomenological surveyability" with the idea that no description can occur in a proof, just rules of grammar.

In fact, Shanker is right about the presence of descriptions in the 4CT proof: they serve to present the programs executed by the computers in the construction of the cases required for the proof of the key reducibility lemma. ${ }^{14}$ Nevertheless, it is our contention that the occurrence of these descriptions in the presentation of the proof is not a sufficient reason for the exclusion of the procedure described from the domain of mathematics - even if we accept Wittgenstein's understanding of mathematical practices as practices in which the use of language is predominantly normative. The elements for denying the thesis according to which the 4CT proof is not a proof but a masked experiment seem to be available in Shanker's own text (for the description of the unavoidable set $U$ is given "together with the operations which the computer has performed"). Let us remember what Wittgenstein has to say about that.

In the Tractatus, Wittgenstein was interest in the sense according to which we can talk about the totality of numbers. This interest was related to the aim of clarifying some confusion that arises when one identifies finite totalities with infinite processes or series ("formal series" in the sense of the applicability of an operation). ${ }^{15}$ Wittgenstein's point is that we can (at least in principle) construct all numbers by means of the successor operation, the "law" generating the series of numbers - this being the only legitimate sense in which we can talk about the infinite totality of the numbers. ${ }^{16}$ Now, although recognizing a lot of changes in the development of Wittgenstein's thought, we believe it is possible to apply these ideas to the case of the 4CT proof, taking the descriptions of the operations performed by the computer as precisely the "manifestation of the law" for the generation of the unavoidable set of reducible configurations; using the vocabulary of proof-theoretical semantics to read Wittgenstein, we could say that what must be surveyable is the description of the effective procedure to execute an operation, not the description of the execution itself. And the description of an operation can function as the rule for its realization. This reading has at least two advantages: we exclude, as Wittgenstein did in latter writings, the appeal to that obscure idea of a "phenomenological surveyability"

\footnotetext{
${ }^{14}$ To be precise, in the first paper the authors refer to results obtained by others for "more detailed descriptions of the algorithms" (Appel Haken 1977a: 431), offering "a general description of the method of defining the discharging procedure" (loc. cit.) in the fifth section of the paper; in the second paper they illustrate "the reducer-choosing algorithms" (Appel, Haken Koch 1977: 493) for the case of configurations with a 12-ring size (a ring size being the number of regions that circumvents a reducible configuration). We are using indifferently "program" and "algorithm" even knowing that the distinction between them is one of the main problems in the philosophy of computer science, as it can be seen in Turner Eden 2009.

${ }^{15}$ See Tractatus 4.1252, 5.232, 6.2 ssq.

${ }^{16}$ For this point see the second chapter of Marion (1998).
} 
Shanker suggested as being still present in the later treatment of surveyability, and it preserves the longstanding distinction between the normative and the descriptive use of language.

Still, if we once more appeal to the distinction between local and global surveyability mentioned above, we could also say that although the 4CT proof is in part locally unsurveyable (for humans but not for the machines!) it is globally surveyable, given exactly the possibility of grasping the rules for proving the lemma that gives the unavoidable set $U$. Of course the plausibility of our reading depends on accepting a functional feature of the descriptions occurring into the proof, i.e., the fact that they can work as a prescription to execute the operations performed by the programs. In this sense we so to speak have control over "the laws" for generating the set $U$. Anyone inclined to implement the calculi by hand could do it, as claimed by Appel and Haken's quote in section two, albeit with the ungrateful result that it would be a boring and de facto impossible task to finish alone in a lifetime. In any case, the person in question, just as the machine, could not be considered as realizing any sort of procedure relevantly similar to a scientific experiment, but just as executing a calculus. To verify if this reading fits with Wittgenstein's approach, we can again remember the tractarian claim that "Calculation is not an experiment". ${ }^{17}$ With this clue in mind, and recognizing the need for clarifying the relation (similarities and dissimilarities) between calculation and proofs in Wittgenstein's philosophy of mathematic, let us deal with the way in which the "characteristic Wittgensteinian invention" (Mühlhölzer 2006: 59), the distinction between proofs and experiments, was explored in more recent discussions about the $4 \mathrm{CT} .^{18}$

After Shanker's explicit introduction of wittgensteinian themes in the controversies about the 4CT proof and his uncomfortable conclusion that Appel and Haken did not prove the 4CT, Shelley Stillwell claimed that we should have a better understanding of the distinction "proof/experiments", before using it to analyze the case of the 4CT proof. For Stillwell (1992), the disputes surrounding the result raised some threats to Wittgenstein's central ideas about mathematics. She intended

\footnotetext{
${ }^{17}$ From the Tractatus onwards, and in a multitude of formulations, Wittgenstein permanently insisted in the distinction between the descriptive nature of the language, predominantly in experimental activities, and the normative character of language at stake in mathematical activities such as calculations and proofs. "Calculation is not an experiment", this sort of slogan stated in the final phrase of a couple of tractarian passages (6.233 and 6.2331), appears in a context in which Wittgenstein is dealing with the question about the need of some kind of intuition in the resolution of mathematical problems - to which he responds that it is the language itself, manipulated in the process of calculating, that brings about this intuition.

${ }^{18} \mathrm{We}$ are emphasizing the idea that this distinction appears late in more recent discussions about the 4CT to stress that its proper wittgensteinian character only began with Shanker's approach to the problem. We can perfectly recognize that Tymoczko's uses the distinction, but given that he did not affiliate himself to Wittgenstein's ideas and, as we considered above, his paper treats the notion of surveyability (the main criterion for the distinction "proof vs. experiment" in its wittgensteinian use) in an ambiguous way, we prefer not to attribute to Tymoczko any strong association to Wittgenstein's perspective.
} 
to defend the pertinence of the dichotomy proof/experiment from Detlefsen and Luker's "attack on Wittgenstein's apriorism". In what follows we will present how Stillwell analyses the concept of surveyability in the context of her approach to the key distinction, through what she calls "[Wittgenstein's] network of logical points of contrast between proofs and experiments." (Stillwell 1992: 133) In our reconstruction, $\mathrm{P}_{n}$ corresponds to logical or conceptual aspects of proofs and $\mathrm{E}_{n}$ to logical or conceptual aspects of experiments. ${ }^{19}$

According to Stillwell, there are five points of comparison between proofs and experiments: whereas $\left(\mathrm{P}_{1}\right)$ proofs transcend the descriptive domain of language, because they introduce new concepts and do not employ genuine propositions, $\left(\mathrm{E}_{1}\right)$ experiments can be completely describable (and understandable) in empirical terms, using genuine propositions ${ }^{20} ;\left(\mathrm{P}_{2}\right)$ when proofs are repeated, the repetition of the result is a necessary condition, which is not the case with experiments, for $\left(\mathrm{E}_{2}\right)$ repeating an experiment implies the possibility of different results; while $\left(\mathrm{P}_{3}\right)$ the construction of a proof does not depend on material conditions, $\left(\mathrm{E}_{3}\right)$ the realization of an experiment depends on precise material conditions ${ }^{21} ;\left(\mathrm{P}_{4}\right)$ proofs can be carried out imaginatively but $\left(\mathrm{E}_{4}\right)$ experiments cannot ${ }^{22}$; and finally, $\left(\mathrm{P}_{5}\right)$ mistakes in calculations nullify the procedure while $\left(\mathrm{E}_{5}\right)$ the same does not occur in experiments. $^{23}$

These points are in internal relation with each other. Let us take as an example of this sort of relation the way Stillwell approaches the link between $\left(\mathrm{P}_{2}\right)-\left(\mathrm{P}_{3}\right)$ and $\left(E_{2}\right)-\left(E_{3}\right)$ - for this will help us to present her understanding of the crucial idea of surveyability. According to Stillwell, while surveyability is a notion stained with epistemological tones, ${ }^{24}$ "holism", $\left(\mathrm{P}_{2}\right)$, is not. ${ }^{25}$ This feature is connected with $\left(\mathrm{P}_{3}\right)$ for to say that proofs are unconditioned also means that they are repeatable

\footnotetext{
${ }^{19}$ Some textual support for each one of these comparison will be given in the footnotes. We will refer to Wittgenstein's Remarks on the Foundations of Mathematics as RFM, indicating as usual the sections and paragraphs, not the pages.

${ }^{20}$ RFM I, Appendix III, 17; III, 29, 31-2, 41; VII, 12, 18.

${ }^{21}$ RFM, III, 55, 65-76.

${ }^{22}$ RFM, I, 98; IV, 4, 6.

${ }^{23}$ RFM III, 68-69.

${ }^{24}$ Stillwell probably inherit this idea from Shanker, who was very much concerned with the elimination of any reference to "medical limitations" in the analysis of Wittgenstein's notion of surveyability, such as not being able to grasp a proof or calculation in one coup d'oeil. This is a point of controversy in the wittgensteinian scholarship milieu. For specific discussions on the notion in Wittgenstein's philosophy of mathematics see Wright (1980), Shanker (1987) and Frascolla (1994). More recent approaches can be found in Mühlhölzer (2006) and Marion (2011).

25 “'A clear proof is reproducible 'as a whole' in that it can be copied, if carefully, without error. But this does not imply that copying a proof entails copying its result. That is, perspicuity does not imply holism. On the other hand, holism does not guarantee perspicuity. The lack of it might make it impossible for us to reproduce a proof, but does not entail that any proof is not holistic. Put otherwise, from the fact that (copying) any proof must include (copying) its result, nothing follows about whether or not that proof is laid clear to view" (Stillwell 1992: 122).
} 
as a whole independently of the context, while, according to $\left(\mathrm{E}_{3}\right)$, experiments are context-dependent. What matters mathematically is that we can only say that we repeat the same proof if we repeat the process and the result - the grammar of "proof" is such that the result is part of its identity. Hence, Stillwell claims that $\left(\mathrm{P}_{3}\right)$ follows from $\left(\mathrm{P}_{2}\right)$ (similarly, we can infer, $\left(\mathrm{E}_{3}\right)$ follows from $\left(\mathrm{E}_{2}\right)$ ). We could say that this implication is the reason why the comparison furnished in $\left(\mathrm{P}_{4}\right)-$ $\left(E_{4}\right)$ obtains: it is only because experiments are settled up to show causal relations occurring in space and time that we cannot reproduce an experiment imaginatively, while we can reproduce a proof "in our heads" - not in the sense of imagining something contingent and non-mathematical, but in the sense of "the impossibility of imagining anything different" (RFM IV, 6). ${ }^{26}$ Returning to the case of the 4CT proof, the main concern Stillwell has with Detlefsen and Luker's account is related to their allegation that every calculus or proof episode contains an eliminable empirical element, introducing in the discussion something she wants to avoid an epistemological aspect:

\begin{abstract}
Detlefsen and Luker are suggesting that a (rational) calculator cannot become convinced that a construction $\mathrm{C}$ establishes result $\mathrm{R}$ unless she/he believes both that no arithmetical errors occur in $\mathrm{C}$ and that, in the pertinent language, the result obtained or found in $\mathrm{C}$ is "R". [ ...] Accordingly, [they] seem right to say that the belief that a specific construction is a proof - if this species of belief is possible - rests on empirical beliefs. In that event, they also would be right that self-sufficient proofs - if proofs be specific constructions must have empirical content. (Stillwell 1992: 125)
\end{abstract}

Stillwell concedes to her opponents that Wittgenstein would not identify the conviction that a construction is a proof with beliefs about specific constructions what would go against $\left(\mathrm{P}_{3}\right)$. Both approaches, Stillwell's and Detlefsen and Luker's, could then be harmonized with regard to the idea that proofs "need nothing outside of itself to be convincing" to use an expression proposed by Tymoczko.

As we already saw, since the Tractatus Wittgenstein credited autonomy of mathematical "propositions" and procedures with respect to reality, in the sense of having no descriptive content like genuine propositions. The problem for Stillwell is that even absorbing the idea of autonomy of proofs, Detlefsen and Luker maintain the identification between calculus or highly calculatorial proofs with experiments. Hence, they generate an insuperable wittgensteinian tension: in what sense an extremely computational proof could not, as a proof, depend on descriptive content or empirical conditions and at the same time be an experimental procedure?

In order to answer this question, Stillwell would have to deal with the notion of calculus or computation such as worked out by Detlefsen and Luker. This notion can be approximated to the heritage of symbolic conceptions of knowledge and mathematics - according to which to calculate is to manipulate signs in symbolic structures, understood as "systems of physical objects subjected to operations of

\footnotetext{
${ }^{26}$ We would like to add: $\left(\mathrm{P}_{6}\right)$ The description of a proof (with some details and in relation with some audiences) is a proof; $\left(\mathrm{E}_{6}\right)$ The description of an experiment never replaces the experiment.
} 
construction and transformation according to rules" (Esquisabel 2012: 21). ${ }^{27}$ Rather, Stillwell prefers to focus on the empirical nature of the allegation that the computer in fact did what it was supposed to do (to execute correctly the calculations programed by Appel, Haken and Koch) suggesting that this can be contemplated by Wittgenstein's "dual" point of view on proofs: in one hand, proofs are practical and variable phenomena (with empirical aspects, typical of matters of fact, such as being subject do material conditions and temporal relations) while in the other they have the force of a standard, being independent of "particular configurations":

\begin{abstract}
Wittgenstein holds that understanding a proof in a sense "transcends" our grasp of specific constructions, for our conviction that such and such is a way of proving $\mathrm{X}$ is not identifiable with, and does not involve, belief about spatial or temporal constructions. Wittgenstein also holds the stronger view that knowledge of proof is independent to a significant degree of beliefs about particular configurations. Still, it is not as if one could learn a proof with no appeal whatever to the latter. In addition, some of the above citations may themselves seem to intimate that "grasping a proof" at least indirectly includes a reference to our knowledge of tokens. (Stillwell 1992: 131-132)
\end{abstract}

In appealing to the process of learning a proof to stress that Wittgenstein recognizes them as acts depending on "particular configurations" Stillwell has made a positive step in relation to Shanker's approach, for she pointed to the growing attention the philosopher gave to mathematics as a human praxis, a knowledge we learn from each other, being trained in the use of mathematical symbols for operations, constructions of "new (conceptual) roads" etc. ${ }^{28}$ The problem seems to be that even highlighting this anthropological aspect of mathematical practices, Stillwell forgets one crucial feature which the philosopher included between the notes on the concept of Übersichtlichkeit - a decisive criterion for the distinction proof versus experiment: the easiness with which a proof is reproduced. In the first paragraph of the third section of RFM Wittgenstein says:

\begin{abstract}
'A mathematical proof must be perspicuous'. Only a structure whose reproduction is an easy task is called a "proof". It must be possible to decide with certainty whether we really have the same proof twice over, or not. The proof must be a configuration whose exact reproduction can be certain. Or again: we must be sure we can exactly reproduce what is essential to the proof. It may for example be written down in two different handwritings or colours. What goes to make the reproduction of a proof is not anything like an exact reproduction of a shade of colour or a handwriting. (RFM, III, 1)
\end{abstract}

On the one hand, Stillwell did not recognize a repeatedly emphasized aspect of the surveyability, that is, the easy reproduction of a surveyable proof; on the other

\footnotetext{
${ }^{27}$ We find in Esquisabel's approach of Leibniz conception of symbolic thought an elegant articulation between local and global surveyability given that amongst the functions of this kind of thought one finds a computational function (identifiable with local surveyability) and a cognitive function (related to the idea that in this kind of thought we grasp the in the syntax of a symbolic system the structures being worked with. This grasping can be associated with global surveyability).

${ }^{28}$ Some discussions Wittgenstein had with his students, including Alan Turing, about the empirical status of calculations during 1939 explores this point (see Wittgenstein 1989).
} 
hand, she deflated the importance of Wittgenstein's emphasis on seeing connections as something fundamental for proofs, stating, for example, that "the time has come to de-emphasize the visual elements in his account" (Stillwell 1992: 132). Now, even if this deflation could serve as a point against Tymoczko - who associates the fact that the 4CT proof cannot be completely visualized (it is not locally surveyable) with it being partially empirical - it is not a good point in interpreting Wittgenstein's discussions on surveyability. In the next section, we intend to show how Stillwell's approach can be complemented, before we resume our narrative of the philosophical significance of the 4CT proof.

\section{In the Third Bank of the River: Concluding Remarks}

In his investigation about the surveyability of mathematical proofs in Wittgenstein's RFM, Mathieu Marion suggests an update of the interpretation of Wittgenstein's "surveyability argument" in the light of the recent studies about visualization in mathematics: "it seems to me", Marion says, "Wittgenstein's point here is not only in line with this recognition of the role of visualization in proofs; he is even using something like it against Russell" (Marion 2011: 152). Now, we would like to follow Marion's hint to question Stillwell's strategy of deflation of the visual character of the surveyability of proofs. But instead of doing this from the perspective of studies such as those in Mancosu (2008), what would really amount to another and different investigation, we would just like to observe that the emphasis Wittgenstein put in the visual aspects of proofs does not correspond to seeing as a merely perceptive capacity, but as the visualization of "what is essential" to the proof - its internal, logical or conceptual relations. We would also like to link this kind of visualization with the idea of global surveyability.

This interpretation can be made plausible with the help of Felix Mühlhölzer's account of Wittgenstein's notion of surveyability as presented in the third part of the RFM. By means of a characterization of surveyability as a purely formal or logical concept, Mühlhölzer proposes an analysis of the concept in terms of four "meaning postulates":

(S1) The surveyability of a proof consists in its possibility of reproduction.

(S2) This reproduction must be an easy task.

(S3) We must be able to decide with certainty whether the reproduction produces the same proof.

(S4) The reproduction of a proof is of the sort of a reproduction of a picture. (Mühlhölzer 2006: 59)

As we saw before, Stillwell's analysis not only considers (S1), but develops an adequate approach to it. Nevertheless, her analysis does not seem to have a place for (S2), as we already mentioned, and this has an important consequence for the discussion on the $4 \mathrm{CT}$ proof, mainly in virtue of a claim made in the first quote of 
this paper, i.e., that the 4CT proof is easily replicable. In order to clarify this claim, we have to consider the context in which Appel presents it

I will use the term pseudo-benign for a proof that satisfies the hand checkability criterion of Thomas Tymoczko, that it be checkable by hand by a single mathematician in a lifetime of work. I will call a proof easily replicable if it makes repeated use of a very small number of straightforward algorithms that are easily programmable and thus are verifiable by an interested reader without overwhelming effort. (Appel 1984: 35, our emphasis)

This is the context of the passage we referred to: "I would argue that our proof of the Four Color Theorem is easily replicable (at least with respect to its computer component) and could also be presented in pseudo-benign form." (loc. cit.) Appel proceeds explaining the main conceptual ideas of the proof and gives some details about how computers were used in it. If one considers that these explanations and details are not sufficient for a good grasp of the logical or conceptual relations in the proof - considering this global survey as insufficient - the thing to do is to read the pair of papers in which the proof is presented and to try to find the sufficient level of detail that could furnish enough understanding of the proof. From a wittgensteinian perspective, though, this local survey (which furnish the description of the operations executed by the algorithms used) could only be of use if it could satisfy (S3), the notion of understanding being here understood not as a psychological but as a formal feature - in this sense, understanding would amount to "being able to operate with symbols" (Mühlhölzer 2006: 64). (S3) then connects with (S4), showing that the synoptic character Wittgenstein associated with the capacity to "know its way about" in a calculus or a proof.

The title of this concluding session naturally leads us to passages 95-99 of $\mathrm{On}$ Certainty (OC) in which Wittgenstein explores the metaphor of the river.

94. But I did not get my picture of the world by satisfying myself of its correctness; nor do I have it because I am satisfied of its correctness. No: it is the inherited background against which I distinguish between true and false.

95. The propositions describing this world-picture might be part of a kind of mythology. And their role is like that of rules of a game; and the game can be learned purely practically, without learning any explicit rules.

96. It might be imagined that some propositions, of the form of empirical propositions, were hardened and functioned as channels for such empirical propositions as were not hardened but fluid; and that this relation altered with time, in that fluid propositions hardened, and hard ones became fluid.

97. The mythology may change back into a state of flux, the river-bed of thoughts may shift. But I distinguish between the movement of the waters on the river-bed and the shift of the bed itself; though there is not a sharp division of the one from the other.

98. But if someone were to say "So logic too is an empirical science" he would be wrong. Yet this is right: the same proposition may get treated at one time as something to test by experience, at another as a rule of testing.

99. And the bank of that river consists partly of hard rock, subject to no alteration or only to an imperceptible one, partly of sand, which now in one place now in another gets washed away, or deposited. 
This splendid and instructive metaphor shows us very important points:

(P1) There are different kinds of propositions in our "system of propositions". But "different" in which sense? A difference "in nature"? A difference "in degree"? According to Wittgenstein, it's a difference in function. Propositions that form our system of propositions can be different according to the function they perform, according to the role they play, in our system of propositions.

(P2) Logic is not an empirical science.

(P3) The function a proposition performs may change: an empirical proposition may be hardened, and hard (logical) propositions may become fluid.

(P4) Some propositions are "subject to no alteration or only to an imperceptible one".

In the case of mathematics, and more specifically in the case of calculations, Wittgenstein employs another interesting metaphor to convey this process of hardening: some propositions may be deposited in the archives.

Proofs and experiments are traditionally conceived as means to establish propositions. But as we have just said, Wittgenstein distinguishes different kinds of propositions according to the function they perform in our system of propositions: empirical propositions and "hardened" propositions. ${ }^{29}$ In the case of empirical propositions, we can think of them as empirical before we conduct an experiment to verify them. But for Wittgenstein, we do not have a mathematical proposition prior to its proof: propositions become "mathematical" because they are proved. But what about the procedures themselves? What about the proofs and the experiments? Are they subjected to the same possibility of variation as the propositions they are thought to establish? Can an experiment become hardened? Can a proof become "fluid"? A good hint to answer this last question would be, as Wittgenstein suggests in conversations with Turing, to think about experiments in didactical situations.

It's clear to us that entering the zone of these metaphors we find ourselves surrounded with a haze, which makes clear vision of the relations between the logical/mathematical and the empirical, the method and the application of the method, and between proofs and experiments, very difficult. But we do think that the proposed functional reading may help to disperse part of the fog and to contribute to the study of these important aspects of some too human practices such as to prove and to make experiments. ${ }^{30}$

\section{References}

Appel, K. (1976, October 21). The proof of the four-color theorem. New Scientist. 154-155.

Appel, K. (1984). The use of the computer in the proof of the four color theorem. Proceedings of the American Philosophical Society, 128(1), 35-39.

Appel, K., \& Haken, W. (1977a). Every planar map is four colorable. Part I: Discharging. Illinois Journal of Mathematics, 21(3), 429-490.

\footnotetext{
${ }^{29}$ For this point see Steiner $(1996,2009)$.

${ }^{30}$ We would like to thank Marcos Silva for organizing the Colloquium in which this paper was first presented and also to $\mathrm{CNPq}$ for funding the research projects that resulted in the paper.
} 
Appel, K., \& Haken, W. (1977b). The solution of the four-color-map problem. Scientific American, 237(4), 108-121.

Appel, K., \& Haken, W. (1986). The four color proof suffices. The Mathematical Intelligencer, $8(1), 10-20$.

Appel, K., Haken, W., \& Koch, J. (1977). Every planar map is four colorable. Part II: Reducibility. Illinois Journal of Mathematics, 21(3), 491-567.

Avigad, G. (2008). Computers in mathematical inquiry. In P. Mancosu (Ed.), The philosophy of mathematical practice (pp. 302-316). Oxford: Oxford University Press.

Bassler, O. B. (2006). The surveyability of mathematical proof: A historical perspective. Synthese, $148,99-133$.

Baker, A. (2016). Non-deductive methods in mathematics. Stanford Encyclopedia of Philosophy. URL http://plato.stanford.edu/entries/mathematics-nondeductive/. ISSN: 1095-5054

Cifoletti, G. (2006). Mathematics and rhetoric. Introduction. Early Science and Medicine, 11(4), 369-389.

Detlefsen, M., \& Luker, M. (1980). The four-color theorem and mathematical proof. The Journal of Philosophy, 77, 803-820.

Esquisabel, O. M. (2012). Representing and abstracting: an analysis of Leibniz's concept of symbolic knowledge. In A. L. Cassanave (Ed.), Symbolic knowledge from Leibniz to Husserl: Studies in logic (Vol. 41, pp. 1-49). London: College Publications.

Frascolla, P. (1994). Wittgenstein's philosophy of mathematics. London/New York: Routledge.

Fritsch, R. \& Fritsch, G. (1998). The four-color theorem. Trad. Julie Peschke. New York/Heidelberg/Berlin: Springer.

Gonthier, G. (2008). Formal proof: The four color theorem. Notices of the American Mathematical Society, 55(11), 1382-1393.

Kreisel, G. (1977). From foundations to science: justifying and unwinding proofs. Recueil des travaux de l'Institut Mathématique - Nouvelle serie (Symposium: Set theory. Foundations of Mathematics, Beograd, 29.08-2.09.1977). Tome 2 (10), pp. 63-72.

Lassale Casanave, A. (2008). Entre la retórica y la dialectica. Manuscrito - Logic Language and Knowledge - Essays on Chateaubriand's Logical Forms, 31(1), 11-18.

Mackenzie, D. (2001). Mechanizing proof: Computer, risk and trust. Cambridge/London: The MIT Press.

Mackenzie, D. (2005). Computing and the cultures of proving. Philosophical Transactions: Mathematical, Physical and Engineering Sciences, 363(1835), 2335-2350. The Nature of Mathematical Proof.

Mancosu, P. (2008). The philosophy of mathematical practice. Oxford: Oxford University Press.

Marion, M. (1998). Wittgenstein, finitism, and the foundations of mathematics. Oxford: Oxford University Press.

Marion, M. (2011). Wittgenstein on the surveyability of proofs. In M. McGinn \& O. Kuusela (Eds.), Oxford handbook of Wittgenstein (pp. 138-161). Oxford: Clarendon Press. ISBN 9780-19-928750-513579108642).

McEvoy, M. (2008). The epistemological status of computer proofs. Philosophia Mathematica, 16, 374-387. (https://doi.org/10.1093/philmat/nkn014).

McEvoy, M. (2013). Experimental mathematics, computers and the a priori. Synthese, 190(3), 397412. https://doi.org/10.1007/s11229-011-0035-1).

Mühlhölzer, F. (2006). A mathematical proof must be surveyable what Wittgenstein meant by this and what it implies. Grazer Philosophische Studien, 71, 57-86. doi:10.1163/18756735071001006 ISSN: 0165-9227 E-ISSN: 1875-6735).

Prawitz, D. (2008). Proofs verifying programs and programs producing proofs. In R. Lupacchini \& G. Corsi (Eds.), Deduction, computation, experiment: Exploring the effectiveness of proof (pp. 81-94). Berlin/Helderberg/New York: Springer.

Robertson, N., Sanders, D., Seymour, P., \& Thomas, R. (1997). The four-color theorem. Journal of Combinatorial Theory, Series B, 70, 2-44.

Saaty, T., \& Kainen, C. (1986). The four-color problem: Assaults and conquest. New York: Dover Publications. 
Secco, G. D. (2015). In a Wittgensteinian light. In H. Heusler, W. de Campos Sanz, \& B. Lopes (Eds.), Why is this a proof? Festschrift for Luiz Carlos Pereira (pp. 45-53). London: College Publications.

Shanker, S. (1987). Wittgenstein and the turning point in the philosophy of mathematics. London: Croom Helm.

Steiner, M. (1996). Wittgenstein: mathematics, regularities, rules. In A. Morton \& S. P. Stich (Eds.), Benacerraf and his critics (pp. 190-212). Oxford: Blackwell Publishing.

Steiner, M. (2009). Empirical regularities in Wittgenstein's philosophy of mathematics. Philosophia Mathematica (III), 17, 1-34.

Stewart, I. (1995). Concepts of modern mathematics. New York: Dover Publications. ISBN 0-48628424-7).

Stillwell, S. (1992). Empirical inquiry and proof. In M. Detlefsen (Ed.), Proof and knowledge in mathematics (pp. 110-134). New York: Routledge.

Swart, E. R. (1980). The philosophical implications of the four-color problem. The American Mathematical Monthly, 87(9), 697-707.

Teller, P. (1980). Computer proof. The Journal of Philosophy, 77, 797-803.

Turner, R., \& Eden, A. (2017). The philosophy of computer science. Stanford Encyclopedia of Philosophy. URL http://plato.stanford.edu/entries/computer-science/. ISSN: 1095-5054)

Tymoczko, T. (1979). The four-color problem and its philosophical significance. The Journal of Philosophy, 27(2), 57-83.

Wang, H. (1981). Popular lectures on mathematical logic. New York: Dover Publications.

Wilson, R. J. (2002). Four colours suffice: How the map problem was solved. London: Penguin Books.

Wittgenstein, L. (1969). In G. E. M. Anscombe \& G. H. Von Wright (Eds.), On certainty (D. Paul \& G. E. M. Anscombe Trans.). New York: Harper Torchbooks.

Wittgenstein, L. (1989). In R. G. Bosanquet, N. Malcolm, R. Rhees, Y. Smythies, \& C. Diamond (Eds.), Wittgenstein's lectures on the foundations of mathematics Cambridge 1939. Chicago: The University of Chicago Press.

Wittgenstein, L. (2004). Tractatus logico-philosophicus (B. F. McGuiness \& D. Pears, Trans.). Routledge, London.

Wright, C. (1980). Wittgenstein on the foundations of mathematics. London: Duckworth. 